\title{
Sizing Up the Most Massive Neutron Star
}

\author{
A satellite experiment has revealed that the heaviest known neutron star \\ is unexpectedly large, which suggests that the matter in the star's inner \\ core is less "squeezable" than some models predict.
}

\section{By Matteo Rini}

N eutron stars are "cosmic zombies"-corpses of massive stars that collapsed in violent explosions after running out of fuel. By studying these ultradense objects, researchers hope to understand the behavior of matter under extreme conditions, which might help in cracking some of the biggest mysteries in physics. As announced at a press conference during the 2021 APS April meeting, NASA's Neutron star Interior Composition Explorer (NICER), an x-ray telescope on the International Space Station, has measured the size of the heaviest known neutron star. The surprisingly large radius measured for this star implies a stiffer-than-expected state of matter in the core, disfavoring models that predict a "squishy" center.

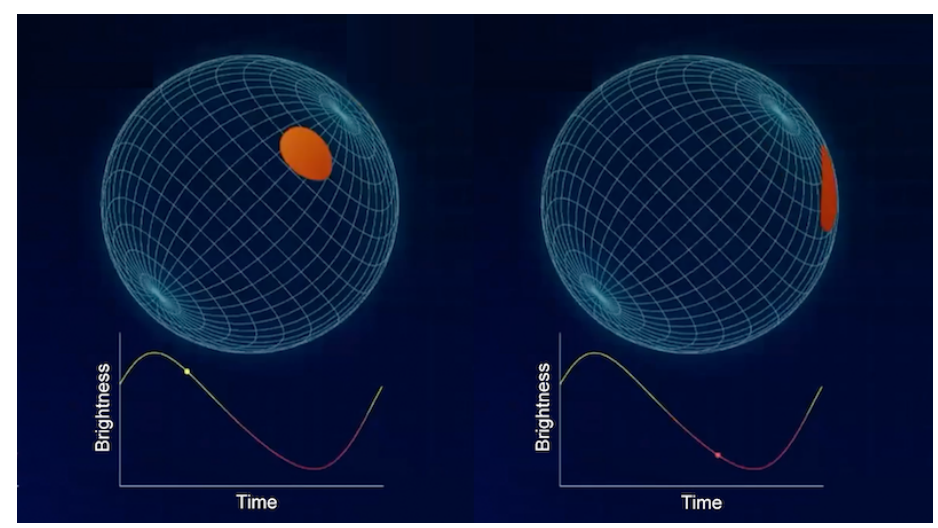

NICER measures the size of a neutron star by tracking the x-ray emission from "hot spots" on the surface as the star rotates. These spots occur at the magnetic poles of the star, where the field slams particles onto the star surface.

Credit: Goddard Space Flight Center
Neutron stars are the densest observable objects in the cosmos-packing twice the mass of the Sun in a sphere as wide as a large city. In the outer core of the star, the large pressure breaks up nuclei into nucleons and crushes protons and electrons together, leaving behind a sea of mostly neutrons. Researchers are unsure, however, about what happens in the inner core of the star. Do neutrons persist or decompose into their quark constituents? Do these quarks interact to form exotic particles?

Since no laboratory experiment can reproduce neutron star conditions, the only option for studying this exceptional state of matter is to observe neutron stars themselves-inferring what's going on inside the star from basic properties such as mass and size. Such measurements, however, are no easy feat. Up until today, about two thousand neutron stars have been discovered, but only a handful of them have been sized up, typically by monitoring the $\mathrm{x}$-ray emission of gas surrounding the star.

NICER has developed a unique sizing method applicable to rapidly rotating neutron stars known as pulsars. As pulsars rotate, hot spots on their surface emit $x$ rays that scan the cosmos like lighthouse beams. The experiment monitors the pulsar's oscillatory $x$-ray brightness, "time stamping" the arrival of each $x$-ray photon with a precision of about $100 \mathrm{~ns}$. The path that these photons take is distorted by the gravitational warping of spacetime around the star, allowing some hot spots to remain visible even as they rotate to the far side of the star.

From the x-ray time-stamped data, the researchers reconstruct the gravitational potential and, in turn, infer the star size. "The 


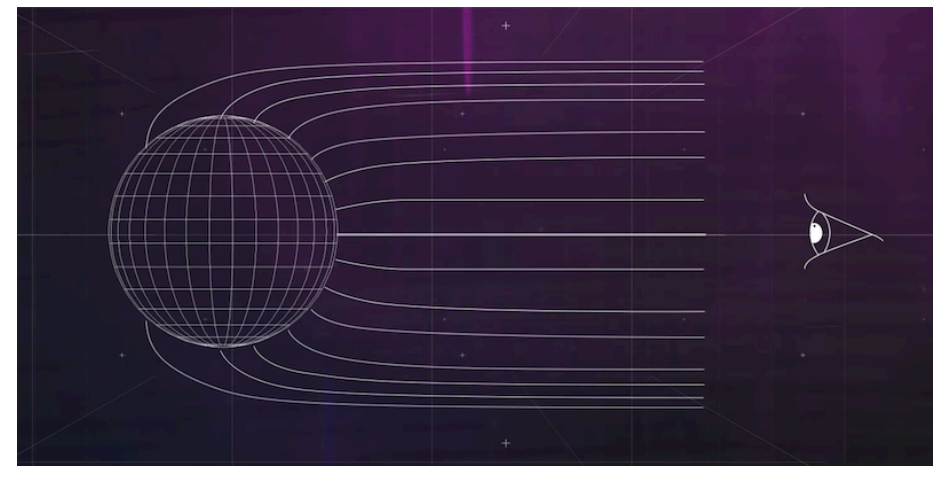

The warping of spacetime near a neutron star redirects light from the star's far side toward us, so that hot spots on the side of the star opposite to the viewer can remain visible.

Credit: Goddard Space Flight Center/Chris Smith (USRA/GESTAR)

combination of $\mathrm{x}$-ray spectroscopy capabilities with timing is a unique feature of NICER," which allows researchers to fully exploit information on the star's spin to constrain its properties, says NICER science lead Zaven Arzoumanian of NASA's Goddard Space Flight Center in Maryland.

The NICER Collaboration first used this method in 2019 to measure PSR J0030, a pulsar 1000 light years from Earth. Weighing 1.4 solar masses, J0030 was found to have a diameter of about $26 \mathrm{~km}$. In the new measurement, the collaboration turned to the most massive known neutron star, PSR J0740, in the "giraffe" constellation. Nearly 4 times more distant than J0030, J0740 is 20 times fainter and was thus a "stretch goal for the experiment," says Arzoumanian. But its mass ( 2.1 solar masses) makes this pulsar "so exceptional" that the team decided to devote a significant amount of time to measuring it, he says.

The collaboration tasked the data analysis to two independent teams, who used different assumptions on, for instance, the $x$-ray background in the sky and the instrument calibration. The teams, led by Anna Watts of the University of Amsterdam and by Cole Miller of the University of Maryland in College Park, came up with similar values for the most likely diameter of the star: 25 and $27 \mathrm{~km}$, respectively, both close to that of the previously measured, lighter pulsar.

"This is a remarkable result," says Sanjay Reddy, a theorist at

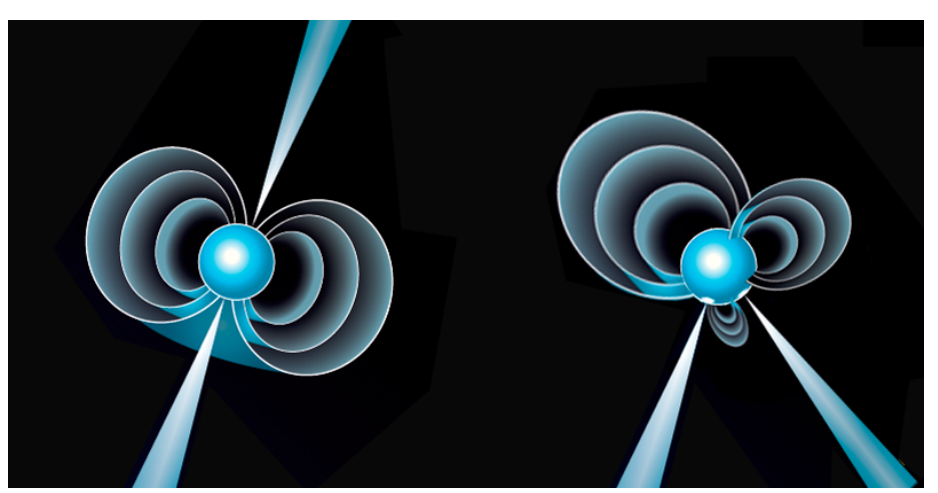

Pulsars are often pictured as perfectly symmetric dipoles (left), emitting beams of radiation from two spots at two opposite poles. In both stars so far targeted by NICER, two hot spots in the same hemisphere suggest a much more complex magnetic field configuration (right).

Credit: Anna Bilous/ASTRON

the University of Washington in Seattle. He says the size measurement will allow researchers to vet different options for the star interior. In some models, neutrons break apart into free-roaming quarks, which leads to a squishy, compressible core. These models deliver the counterintuitive prediction that neutron stars should become smaller as their mass increases. Other models, in which some neutrons persist, or the quarks interact strongly, predict a harder-to-compress form of matter. The similar diameters found for J0030 and J0740 "strongly disfavors the squishiest models," says Reddy.

Together, the two measurements of J0740 and J0030 offer another thought-provoking conclusion, says Watts. In textbooks, pulsars are depicted as perfectly symmetric magnetic dipoles, with their hot spots at each of the pulsar's poles. For both stars investigated by NICER, however, the hot spots appear to lie on the same hemisphere, suggesting a much more complex and asymmetric field configuration. Having seen this asymmetry in the first two stars where the hot spots have been mapped, "this beautiful dipole cartoon of pulsars is likely wrong," says Watts.

With NICER setting out to measure more pulsars, and with the prospects of seeing many neutron star mergers through gravitational-wave detection, we are entering a "Golden Age" for neutron star physics, says Reddy. He says that 
understanding the nature of matter inside these stars will have implications on many fundamental questions, as neutron stars play a role in the synthesis of the heaviest elements, and they are promising targets for spotting dark matter.
Matteo Rini is the Editor of Physics. 